\title{
Nasal bone in screening for T21 at 11-13 + 6 weeks of gestation - a multicenter study
}

\author{
Bartosz Czuba' ${ }^{1}$, Dawid Serafin ${ }^{1}$, Piotr Węgrzyn ${ }^{2}$, Wojciech Cnota ${ }^{1}$, Mariusz Dubiel $^{3}$, \\ Marek Mączka ${ }^{4}$, Dariusz Zarotyński ${ }^{5}$, Aleksandra Ruci ${ }^{1}$, Mirosław Wielgoś6 \\ Krzysztof Sodowski ${ }^{1}$, Dariusz Borowski ${ }^{6}$ \\ ${ }^{1}$ Teaching Department of Obstetrics and Gynecology in Ruda Slaska, Medical University of Silesia, Poland \\ ${ }^{2}$ Department of Obstetrics and Perinatology, Medical University of Warsaw, Poland \\ ${ }^{3}$ Department of Maternal-Fetal Medicine and Gynecology. Chair of Obstetrics, Bydgoszcz, \\ The Nicolaus Copernicus University in Toruń, Collegium Medicum UMK in Bydgoszcz, Poland \\ ${ }^{4}$ Gynecological-Obstetrics Hospital in Opole, Poland \\ ${ }^{5}$ The R. Czerwiakowski Gynecological-Obstetrics Hospital in Krakow, Poland \\ ${ }^{6} 1^{\text {st }}$ Department of Obstetrics and Gynecology, Medical University of Warsaw, Poland
}

\begin{abstract}
Objectives: Trisomy 21 is one of the most common chromosomal defects diagnosed prenatally. Screening for Down syndrome is based on maternal age, measurement of crown-rump length, nuchal translucency and fetal heart rate, together with free $\beta$-hCG and PAPP-A at 11 to $13+6$ weeks. Introduction of additional ultrasound marker of trisomy 21 (evaluation of the nasal bone) may result in increased DR and decreased invasive diagnostic testing rates (FPR).

Material and methods: Ultrasound scan with NB evaluation was performed in 5814 fetuses during routine screening for chromosomal defects at 11 to $13+6$ weeks of gestation. DR and FPR coefficients were calculated for 4 levels of risk as cut-off points for screening model 1, based on MA, NT, and first trimester biochemistry, as well as for screening model 2 , based on MA, NT, first trimester biochemistry and NB.

Results: There were 5708 normal cases, 71 cases of trisomy 21 and 35 cases of other chromosomal defects. NB was absent in $46(64.8 \%)$ cases and present in 25 (35.3\%) cases of trisomy 21, comparing to present NB in 5463 (95.7\%) and absent in 245 (4.3\%) of normal cases.

Conclusions: First-semester screening with additional NB assessment significantly increases the detection rate for trisomy 21 and decreases the rate of false-positive results. Adding NB evaluation at the risk level of 1:50 causes only a small increase in detection rate. Invasive procedures should be performed in that group regardless NB assessment.
\end{abstract}

Key words: nasal bone, chromosomal defects, screening for trisomy 21

Ginekologia Polska 2016; 87, 11: 751-754

\section{INTRODUCTION}

Trisomy 21 (Down syndrome) is one of the most common chromosomal defects diagnosed prenatally [1]. Phenotypic characteristics of this disease were described by John Langdon Down in 1866 [2], and are currently used as markers in prenatal ultrasound [3]. Absent nasal bone (NB) is a frequent phenotypic defect in fetuses with trisomy 21 (T21) and affects approximately $70 \%$ of fetuses with T21 and 1-3\% of fetuses with normal karyotype [1,4,5]. Thus, evaluation of
NB presence may constitute an additional marker to increase the efficacy of screening for trisomy 21 [6].

At present, assessment of nuchal translucency (NT), maternal age (MA), and fetal heart rate (FHR), together with first trimester biochemistry $(\mathrm{BC})$ [evaluation of the concentration of free beta subunit of human chorionic gonadotropin (free $\beta$-hCG) and pregnancy-associated plasma protein A, (PAPP-A) in maternal blood], constitute a screening standard for Down syndrome at 11 to 13 weeks and 6 days of gesta- 


\begin{tabular}{|c|c|c|c|c|c|c|c|}
\hline Variable & Mean & SD & Min. & Median & Max. & $\begin{array}{c}\mathbf{2 5} \% \\
\text { percentile }\end{array}$ & $\begin{array}{c}\mathbf{7 5} \% \\
\text { percentile }\end{array}$ \\
\hline Maternal age (years) & 32.04 & 5.38 & 14.00 & 33.00 & 46.00 & 28.00 & 36.00 \\
\hline CRL [mm] & 63.54 & 8.29 & 45.00 & 63.30 & 84.00 & 57.77 & 69.00 \\
\hline NT [mm] & 1.86 & 0.73 & 0.80 & 1.80 & 15.00 & 1.50 & 2.00 \\
\hline FHR (BPM) & 160.41 & 16.58 & 130 & 160 & 196 & 156 & 164 \\
\hline Free $\beta$-hCG MoM & 1.32 & 0.90 & 0.125 & 1.07 & 7.68 & 0.73 & 1.66 \\
\hline PAPP-A MoM & 1.01 & 0.57 & 0.115 & 0.90 & 6.00 & 0.61 & 1.28 \\
\hline
\end{tabular}

tion [6]. This scheme is characterized by a high (approximately $90 \%$ ) detection rate (DR) of chromosomal defects, mainly T21, with a 5\% False Positive Rate (FPR) $[5,7]$.

Introduction of additional ultrasound markers of trisomy 21 (evaluation of the nasal bone, as well as assessment of tricuspid and ductus venosus blood flow) results in increased DR (up to $95 \%$ ) and decreased invasive diagnostic testing rates (FPR) [8].

\section{MATERIAL AND METHODS}

Ultrasound scan with NB evaluation was performed in 5814 fetuses during routine screening for chromosomal defects at 11-13+6 weeks of gestation. The test was carried out using the Voluson Expert VE 730 and Voluson Expert E8 by sonographers with the FMF (Fetal Medicine Foundation) certificate, confirming their skills to evaluate fetuses at 11-13+6 wks. The following were investigated: CRL (Crown-rump length), MA, NT, and FHR (Table 1). In addition to the ultrasound examination, free $\beta-h C G$ and PAPP-A levels were measured in maternal blood. The results of the biochemical test, expressed as multiples of the median (MoMs), were also included in the study. This retrospective, cross-sectional, descriptive, non-interventional study used anonymized data, hence the study protocol did not require ethical approval under local rules.

NB was routinely assessed in each patient with the image of the fetal head in the sagittal plane enlarged to include only the profile and the upper part of the chest (Figure 1). All criteria required by the Fetal Medicine Foundation for imaging were met during the examination $[6,9,10]$. CRL, MA, NT, FHR, free $\beta$-hCG and PAPP-A values were entered into the Astraia software to assess the risk for trisomy 21.

In the high-risk group amniocentesis for karyotyping was performed. In remaining cases, neonatologists phenotypically evaluated newborns for follow-up.

DR and FPR coefficients were calculated for 4 levels of risk as cut-off points for screening model 1, based on MA, NT, and first trimester biochemistry (Table 2), as well as screening model 2, based on MA, NT, first trimester biochemistry, and NB (Table 3).

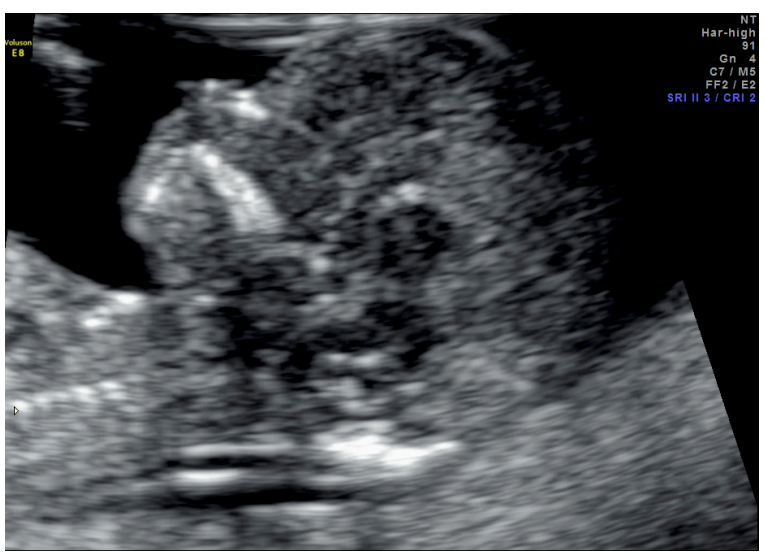

Figure 1. Routine assessment of the nasal bone with the image of the fetal head in the sagittal plane enlarged to include only the profile and the upper part of the chest. Present NB

Table 2. Comparison of DR and FPR in screening model 1 (without NB)

\begin{tabular}{|l|c|c|c|c|}
\hline Risk cut-off for trisomy $\mathbf{2 1}$ & $\mathbf{1 : 3 0 0}$ & $\mathbf{1 : 2 0 0}$ & $\mathbf{1 : 1 0 0}$ & $\mathbf{1 : 5 0}$ \\
\hline DR [\%] & 84.5 & 83.1 & 80.3 & 78.8 \\
\hline FPR [\%] & 10.7 & 8.5 & 5.7 & 3.6 \\
\hline
\end{tabular}

Table 3. Comparison of DR and FPR for screening model 2 (with NB assessment)

\begin{tabular}{|l|c|c|c|c|}
\hline Risk cut-off for trisomy $\mathbf{2 1}$ & $\mathbf{1 : 3 0 0}$ & $\mathbf{1 : 2 0 0}$ & $\mathbf{1 : 1 0 0}$ & $\mathbf{1 : 5 0}$ \\
\hline DR [\%] & 93.0 & 91.5 & 90.1 & 80.3 \\
\hline FPR [\%] & 2.0 & 2.0 & 1.9 & 1.5 \\
\hline
\end{tabular}

All generated data were used to verify the usefulness of NB evaluation as the component of the screening test. The results were analyzed with PQStat statistical package, version 1.4.2.324.

CRL, MA, NT, free ß-hCG MoM, PAPP-A MoM, and FHR were analyzed in each group with $U$ Mann-Whitney test in relation to karyotype. The presence of NB in the investigated groups was measured with chi-squared test. Maternal age ( $\geq 35$ years), T21 NT risk ( $\geq 1: 300)$, T21 BC 
risk ( $\geq 1: 300)$, and T21 BC NT risk ( $\geq 1: 300$ ) were compared in both groups using cross tabulation and chi-squared test in relation to karyotype. The sensitivity and FPR were determined. ROC analysis for both models ( 1 and 2) of T21 risk was performed. The $p$-value of $<0.05$ was considered statistically significant.

\section{RESULTS}

There were 35 cases of other chromosomal defects that were excluded from the study ( 18 of trisomy 18,6 of trisomy 13,5 of Turner syndrome, 3 of chromosomal inversion, 2 of triploid and 1 of trisomy 9). The presence and absence of NB was analyzed only in trisomy 21 and normal cases. There were 71 cases of trisomy 21 (1.22\%) and 5708 normal cases. NB was absent in $46(64.8 \%)$ cases (Figure 2), and present in 25 (35.3\%) cases of trisomy 21, comparing to present NB in 5463 (95.7\%) and absent in 245 (4.3\%) of normal cases (Table 4). Study group description is presented in Table 1.

In screening model 1 (without NB) for the cut-off of 1:300, DR was $84.5 \%$ and FPR was $10.7 \%$. When the additional marker (NB) was added to screening (model 2), at cut-off of 1:300, DR increased to $93.0 \%$ for $2.0 \%$ FPR. For the cut-off of $1: 100$, DR was $80.3 \%$ for $5.7 \%$ FPR without NB assessment. After adding NB, DR increased to $90.1 \%$ at $1.9 \%$ FPR. Both screening models were also compared at cut offs of 1:200 and 1:50 (Tables 2 and 3).

Comparison of screening models 1 and 2 and was also performed with the use of the ROC analysis (Figures 3 and 4).

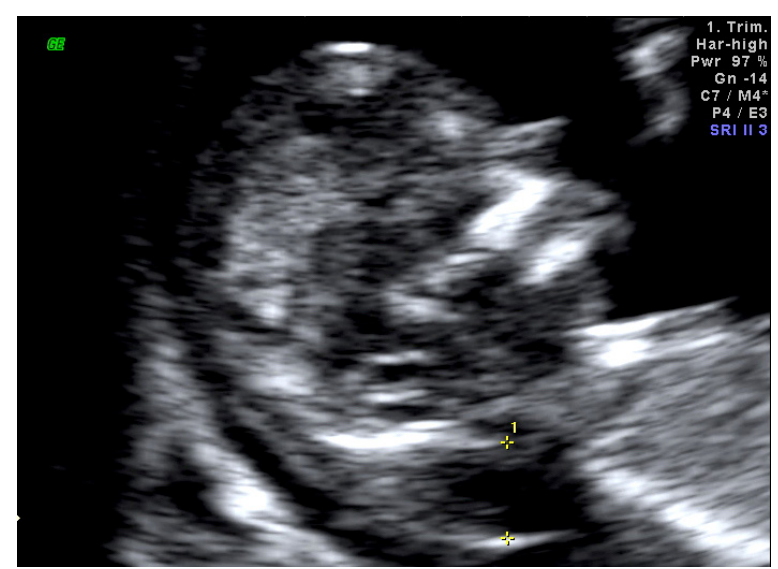

Figure 2. Nasal bone is less echoic that overlying skin, hence classified as "absent"

\begin{tabular}{|l|c|c|c|}
\hline Table 4. Presence and absence of the nasal bone \\
\hline & $\begin{array}{c}\text { Total } \\
\text { number }\end{array}$ & $\begin{array}{c}\text { Present NB } \\
(\%)\end{array}$ & $\begin{array}{c}\text { Absent NB } \\
(\%)\end{array}$ \\
\hline Normal neonates & 5708 & $5463(95.7 \%)$ & $245(4.3 \%)$ \\
\hline Trisomy 21 cases & 71 & $25(35.2 \%)$ & $46(64.8 \%)$ \\
\hline
\end{tabular}

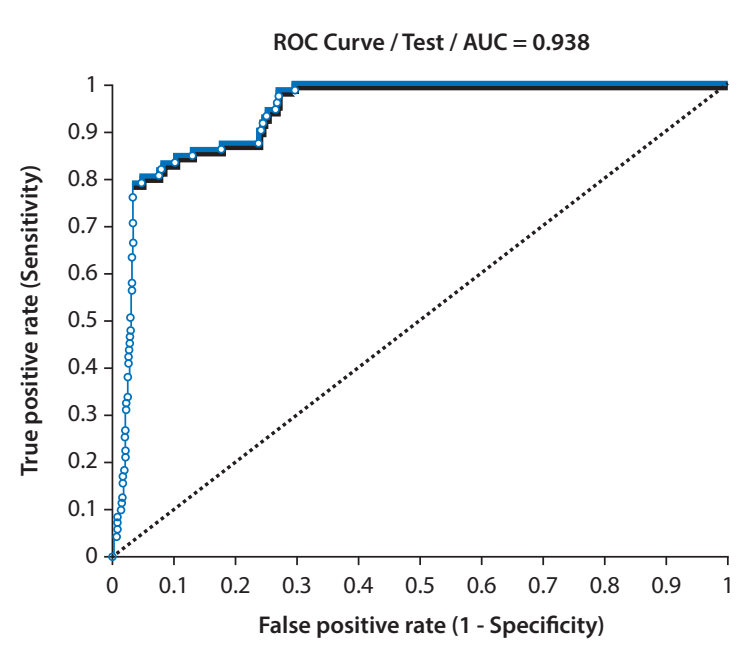

Figure 3. ROC analysis for model 1

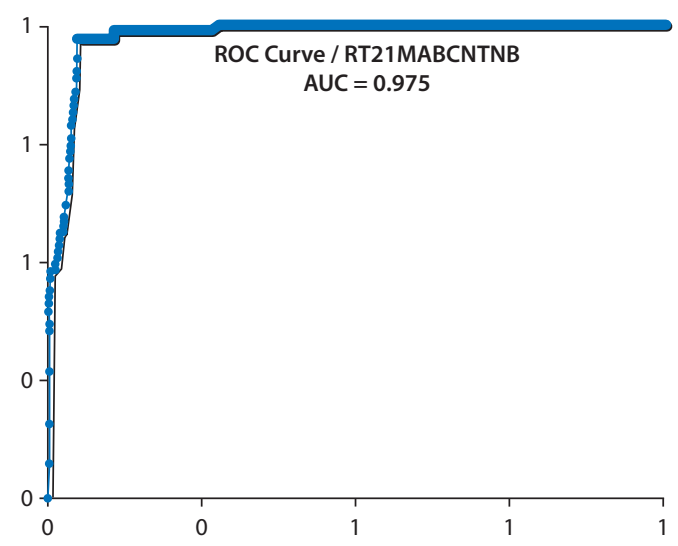

Figure 4. ROC analysis for model 2

\section{DISCUSSION}

Absent or hypoplastic NB is one of the characteristic features of trisomy 21. NB may be evaluated on ultrasound at $11-13+6$ weeks. It is visualized as a thin line, more echogenic than the overlying skin. NB assessment has been applied to optimize screening for trisomy 21 [8, 9]. In 2001, Cicero et al., proved an important correlation between absent NB and the presence of trisomy 21 in their study on over 3800 fetuses [4]. The results of a study by Orlandi et al., who also investigated the effect of NB evaluation during routine first-trimester screening, are comparable with our findings. These authors assessed over 2300 fetuses and found 15 cases with Down syndrome, including 8 with absent NB. During statistical analysis, a comparison of the results with the risk cut-off level of 1:250 for standard screening was performed and revealed DR and FPR of $87 \%$ and $4.3 \%$, respectively, and of $90 \%$ and $2.5 \%$, respectively with additional NB evaluation [10]. Zoppi et al., investigated NT and NB in 5532 fetuses [1], and found absent NB in 70\%, 80\% and $66 \%$ of the fetuses with Down's, 
Edwards and Turner's syndromes, respectively, as well as $0.2 \%$ of fetuses with normal karyotype [1]. According to Otano et al. [11], agenesis of the nasal bone is diagnosed in $60 \%$ of T21 and $0.6 \%$ of healthy fetuses, what is consistent with our findings. In our study, 71 cases with Down syndrome were confirmed, including 46 (64.7\%) with absent NB.

In 2006, Cicero et al. proved in another study that combination of NB evaluation with other basic parameters of the first-trimester screening decreases the FPR by half, from $5 \%$ to $2.5 \%$ for cut-off level of 1:100 [5], which in similar to our findings (FPR decreased from $5.7 \%$ to $1.9 \%$ for cut-off level of 1:100).

Also in 2006, Kozlowski et al. studied approximately 300 fetuses and found no significant advantages of including NB evaluation into the first-semester routine screening. In fact, sensitivity was higher when that marker was excluded from the calculations. According to these authors, DR with and without NB evaluation was $77.8 \%$ and $94.4 \%$, respectively for cut-off level of 1:300 [12]. Our data showed higher efficacy (DR of 84.5 and $93.0 \%$ respectively), with low rates of false-positive results (FPR of 10.7 and 2.0\% respectively). Possibly, divergent findings were the result of a small sample in the paper of Kozlowski et al.

Our prospective study demonstrates that NB is absent in approximately $4 \%$ of the fetuses with normal karyotype and $65 \%$ of the fetuses with Down syndrome at $11-13+6$ weeks. Effective first-semester screening is based on MA, NT, FHR, CRL as well as maternal levels of $\beta$ hCG and PAPPA-A, resulting in FPR of $5.7 \%$ with risk cut-off of $1: 100$. Inclusion of NB into the screening test reduced FPR to $1.9 \%$ without the necessity to change the cut-off level. That allows to lower the number of invasive tests, and consequently of iatrogenic miscarriages and the related costs. Kagan et al., assessed the usefulness of NB evaluation as an additional marker of chromosomal defects (trisomy 21, 18, and 13) [13].

The suggested risk division into the cut-offs of 1:50, $1: 100,1: 200$ and 1:300 demonstrated the usefulness of NB as an additional marker of trisomy 21 in each group. Similar observations were made in our study. Owing to a high rate of trisomy 21 in the 1:50 risk group, invasive diagnostic testing is immediately advised in these patients. In case of risk lower than 1:50 NB marker is applicable due to significantly decreased FPR and higher DR [13]. Our study showed increased DR (from 78.7\% to $80.3 \%$ ) and lower FPR in each risk group (from $3.6 \%$ to $1.5 \%$ ), depending on the model (1 or 2). Comparisons of models 1 and 2 at the risk level of 1:50 revealed a slightly higher DR and significantly lower number of indications for invasive testing.

The FMF management protocol recommends invasive diagnosis for trisomy 21 risk $\geq 1: 50$, regardless of the presence of the NB, similarly to other additional ultrasound markers (for example fronto-maxillary facial angle evaluation) [14]. The sonographer assessing the fetal nasal bone has to have an appropriate experience. Lack of appropriate training, certification and auditing has significant negative impact of the FPR [15]. Proper ultrasound evaluation of ultrasound markers of chromosomal defects, including the nasal bone, should lead to more selective referral to invasive testing, and, as a consequence, fewer procedure-related complications and pregnancy losses.

\section{CONCLUSIONS}

First-semester screening with additional NB assessment significantly increases the detection rate for trisomy 21 and decreases the rate of false-positive results.

Adding NB evaluation at the risk level of $\geq 1: 50$ (based on ultrasound and first trimester biochemistry) causes only a small increase in detection rate. Invasive procedures should be performed in that group regardless NB assessment.

\section{Conflict of interest}

Authors don't have any sources of financial support to disclosure.

\section{REFERENCES}

1. Zoppi MA, Ibba RM, Axiana C, [et al.]. Absence of fetal nasal bone and aneuploidies at first-trimester nuchal translucency screening in unselected pregnancies. Prenat Diagn. 2003, 23, 496-500.

2. Down L. Observations on an ethnic classification of idiots. Clin Lectures and Reports, London Hospital, 1866, 259-262.

3. Wright D, Syngelaki A, Bradbury I, [et al.]. First-trimester screening for trisomies 21, 18 and 13 by ultrasound and biochemical testing. Fetal Diagn Ther. 2014, 35, 118-126.

4. Cicero S, Curcio P, Papageorghiou A, [et al.]. Absence of nasal bone in fetuses with trisomy 21 at 11-14 weeks of gestation: an observational study. Lancet. 2001, 358, 1665-1667.

5. Cicero S, Avgidou K, Rembouskos G, [et al.]. Nasal bone in first-trimester screening for trisomy 21. Am J Obstet Gynecol. 2006, 195, 109-114.

6. Kagan KO, Staboulidou I, Cruz J, [et al.]. Two-stage first-trimester screening for trisomy 21 by ultrasound assessment and biochemical testing. Ultrasound Obstet Gynecol. 2010, 36, 542-547.

7. Nicolaides $\mathrm{KH}$, Spencer K, Avgidou K, [et al.]. Multicenter study of first-trimester screening for trisomy 21 in 75821 pregnancies: results and estimation of the potential impact of individual risk-orientated two-stage first-trimester screening. Ultrasound Obstet Gynecol. 2005, 25, 221-226.

8. Illa M, Mula R, Arigita M, [et al.]. Likelihood ratios to apply for nasal bone, ductus venosus and tricuspid flow at the 11-13 weeks' scan in Down syndrome screening. Fetal Diagn Ther. 2013, 34, 116-120.

9. Sonek J. First trimester ultrasonography in screening and detection of fetal anomalies. Am J Med Genet C Semin Med Genet. 2007, 145C, 45-61.

10. Orlandi F, Rossi C, Orlandi E, [et al.]. First-trimester screening for trisomy-21 using a simplified method to assess the presence or absence of the fetal nasal bone. Am J Obstet Gynecol. 2005, 192, 1107-1111.

11. Otano $\mathrm{L}$, Aiello $\mathrm{H}$, Igarzabal $\mathrm{H}$, [et al.]. Association between first trimester absence of fetal nasal bone on ultrasound and Down syndrome. Prenat Diagn. 2002, 22, 930-932.

12. Kozlowski P, Knippel AJ, Froehlich S, [et al.]. Additional performance of nasal bone in first trimester screening. Ultraschall Med. 2006, 27, 336-339.

13. Kagan $\mathrm{KO}$, Cicero S, Staboulidou I, [et al.]. Fetal nasal bone in screening for trisomies 21, 18 and 13 and Turner syndrome at 11-13 weeks of gestation. Ultrasound Obstet Gynecol. 2009, 33, 259-264.

14. CnotaW, Borowski D, Wloch A, [et al.]. Pomiar kąta twarzowo-szczekowego u płodów pomiędzy $11+0$ a 13 +6. tygodniem ciąży. Zastosowanie w diagnostyce prenatalnej trisomii 21. Ginekol Pol. 2013, 84, 624-629.

15. Staboulidou I, Wustemann M, Vaske B, [et al.]. Interobserver variability of the measurement of fetal nasal bone length between $11+0$ and 13 +6 gestation weeks among experienced and inexperienced sonographers. Ultraschall Med. 2009, 30, 42-46. 